\title{
Impact of the Tumor Microenvironment on the Gene Expression Profile in Papillary Thyroid Cancer
}

\author{
Malgorzata Oczko-Wojciechowska ${ }^{a}$ Aleksandra Pfeifer ${ }^{a}$ Michal Jarzab ${ }^{b}$ \\ Michał Swierniak $^{c}$ Dagmara Rusinek ${ }^{\mathrm{a}}$ Tomasz Tyszkiewicz $^{\mathrm{a}}$ \\ Malgorzata Kowalska ${ }^{a}$ Ewa Chmielik $^{d}$ Ewa Zembala-Nozynska ${ }^{d}$ \\ Agnieszka Czarniecka $^{e}$ Barbara Jarzab $^{f}$ Jolanta Krajewska ${ }^{f}$
}

\begin{abstract}
${ }^{a}$ Genetic and Molecular Diagnostics of Cancer Department, M. Sklodowska-Curie National Research Institute of Oncology Gliwice Branch, Gliwice, Poland; 'b Breast Unit, M. Sklodowska-Curie National Research Institute of Oncology Gliwice Branch, Gliwice, Poland; 'C Center of New Technologies, University of Warsaw, Warsaw, Poland; dTumor Pathology Department, M. Sklodowska-Curie National Research Institute of Oncology Gliwice Branch, Gliwice, Poland; e Oncologic and Reconstructive Surgery Clinic, M. Sklodowska-Curie National Research Institute of Oncology Gliwice Branch, Gliwice, Poland; ${ }^{f}$ Nuclear Medicine and Endocrine Oncology Department, M. Sklodowska-Curie National Research Institute of Oncology Gliwice Branch, Gliwice, Poland
\end{abstract}

\section{Keywords}

Papillary thyroid cancer · Tumor microenvironment · Gene expression profile $\cdot$ Tumor stroma

\begin{abstract}
Transcriptome of papillary thyroid cancer (PTC) is well characterized and correlates with some prognostic and genotypic factors, but data addressing the interaction between PTC and tumor microenvironment (TME) are scarce. Therefore, in the present study, we aimed to assess the impact of TME on gene expression profile in PTC. We evaluated the gene expression profile in PTC and normal thyroid cells isolated by laser capture microdissection and in whole tissue slides corresponding to the entire tumor. We included 26 microdissected samples for gene expression analysis (HGU133 PLUS 2.0, Affymetrix, currently Thermo Fisher Scientific USA): 15 PTC samples, 11 samples of normal thyrocytes,
\end{abstract}

and 30 whole slides (15 PTC and 15 normal thyroid). Transcripts were divided into three groups: differentially expressed both in microdissected and whole slides, transcripts differently expressed in microdissected samples and not changed in whole slides, and transcripts differentially expressed in whole slides and not changed in microdissected samples. Eleven genes were selected for validation in an independent set of samples; among them, four genes differentiated only microdissected PTC and normal cells. Two genes (PTCSC and CTGF) were confirmed. One gene (FOS) was not confirmed by the validation, whereas $E G R 1$ was also significant in whole slide analysis. The other seven genes (TFF3, FN1, MPPED2, MET, KCNJ2, TACSTD2, and GALE) showed differentiated expression in microdissected thyrocytes and in whole tumor slides. Most of identified genes were related to the tumor-microenvironment interaction and confirmed the crosstalk between TME and cancer cells.

(c) 2020 S. Karger AG, Basel

Malgorzata Oczko-Wojciechowska

Genetic and Molecular Diagnostics of Cancer Department

M. Sklodowska-Curie National Research Institute of Oncology Gliwice Branch

Wybrzeza AK 15, PL-44-100 Gliwice (Poland)

malgorzata.oczko-wojciechowska@io.gliwice.pl 


\section{Introduction}

Papillary thyroid cancer (PTC) is the most common type of differentiated thyroid cancer deriving from the follicular cell of the thyroid gland, accounting for about $80 \%$ of all thyroid malignancies [1]. Over a decade, highthroughput methods, including next-generation sequencing and microarrays, have been used for the evaluation of PTC genotype and gene expression profile to find characteristic features related to neoplastic transformation and turn it into diagnostic and clinically valuable information [2-5]. The analysis of 500 PTC tumors, based on next-generation sequencing, under the genotyping project Cancer Genome Research Network, confirmed four major molecular events present in the majority of PTC cases: $B R A F^{\mathrm{V} 600 \mathrm{E}}, R A S$, and TERT point mutations as well as RET/PTC rearrangements [4]. Although the PTC transcriptome is well characterized $[3,6-8]$ and correlates with some prognostic and genotypic factors $[3,4]$, biological mechanisms of neoplastic transformation in PTC are not unequivocally elucidated. Nevertheless, these data do not explain the whole biology of PTC tumorigenesis and are only to some extent helpful in diagnostics (BRAF, TERT, RET/PTC rearrangements) [9-14] and prognosis (BRAF, TERT) [15-18].

It is well known that the tumor microenvironment (TME) contributes to cancer development and promotes angiogenesis, invasion, metastasis, and chronic inflammation [19]. The possible role of TME in response to chemotherapy [20] or antiangiogenic therapy [21] has been addressed recently. In healthy tissues the wound healing process is promoted by the activation, migration, and proliferation of fibroblasts, whereas cancer-associated fibroblasts play a role in tumor growth and therefore are crucial for disease progression [19, 22].

Already published data which addressed the interaction between PTC and stromal tissue, lymphocytic infiltrate, or normal thyrocytes and their influence on gene expression profile are scarce. The results of our previous analysis demonstrated a strong impact of stromal cells on PTC gene expression profile. We observed that immunity-related genes provided the most intensive confounding signal, probably related to lymphocyte tumor infiltration [3]. The proportion of particular cells may vary between tumors, which may also change the pattern of a transcriptome analyzed in whole tissue slide.

In the present study, we aimed to assess the impact of TME on gene expression profile in PTC. To achieve this goal, we decided to evaluate the gene expression pattern of PTC and normal thyroid cells, isolated by laser capture microdissection and in whole tissue slides. We believe that such an approach allows for wider analyses and, in consequence, for better understanding of the biology of PTC.

\section{Materials and Methods}

\section{Analysis of PTC Transcriptome}

Tumor tissues samples were removed during surgical resection, snap-frozen on dry ice, and stored at $-80^{\circ} \mathrm{C}$ until use.

Fifteen microdissected PTC samples, 11 samples containing normal thyrocytes taken from contralateral thyroid lobe and 15 pairs of whole slide PTC/macroscopically unchanged tissue, were subjected to transcriptome profiling with oligonucleotide microarrays HG-U133 PLUS 2.0 (Affymetrix, currently Thermo Fisher Scientific USA). Total RNA was extracted from $1 \times 10^{6}$ $\mu \mathrm{m}^{3}$ of microdissected tissue (PALM system) and extracted by RNeasy Micro Kit (Qiagen GmbH, Hilden, Germany). Ten to thirty nanograms of total RNA were used for double round amplification (Two Cycle Target Labeling Kit, Affymetrix/Thermo Fisher, USA). From the whole slides 100 ng of total RNA were used for one round amplification (GeneChip 3' IVT Express Kit, Affymetrix/Thermo Fisher, USA). All reactions, washing, staining, and scanning were performed according to the manufacturer's manual.

Validation of Obtained Results by Quantitative Real-Time Polymerase Chain Reaction

RNA subjected to quantitative real-time polymerase chain reaction (qRT-PCR) validation was extracted from 31 pairs of whole slide PTC/normal thyroid, 12 microdissected PTC samples, 6 samples of microdissected normal thyrocytes, 12 PTC slides, and 13 whole normal thyroid slides.

Total RNA was extracted using RNeasy Mini Kits (Qiagen $\mathrm{GmbH}$ ) according to the manufacturer's protocol. Reverse transcription reaction was performed using Qiagen SensiScript RT Kits and $5 \mathrm{ng}$ of input RNA according to the manufacturer's protocol. All genes were amplified by the $7900 \mathrm{HT}$ Fast Real-Time PCR system (Life Technologies/Thermo Fisher, USA). For amplicon design, Roche Universal Probe Library was used. For normalization of the qRT-PCR data, EIF3S10, HADHA, and UBE2D2 genes were used. The normalization factor was obtained using the GeNorm applet for Microsoft Excel.

\section{Statistical Analysis}

Microarray Data Analysis. All bioinformatic analyses of gene expression profile were performed in an R/Bioconductor environment. The GCRMA algorithm was used for normalization. Unsupervised analysis was perform using principal component analysis. Supervised analysis was based on gene expression comparison using Student $t$ test. Differentiated transcripts were selected in three separate comparisons: (1) transcripts differentially expressed in microdissected and whole slides - nominal $p<0.001(\mathrm{FDR}<2 \%)$ and fold change $>4$ (both directions); (2) transcripts differently expressed in microdissected samples (criteria as above) and not changed in whole slides - nominal $p>0.01$ (FDR $>5 \%)$; (3) transcripts differentially expressed in whole slides and not changed in microdissected samples (criteria are given in point 2). Hypergeo- 


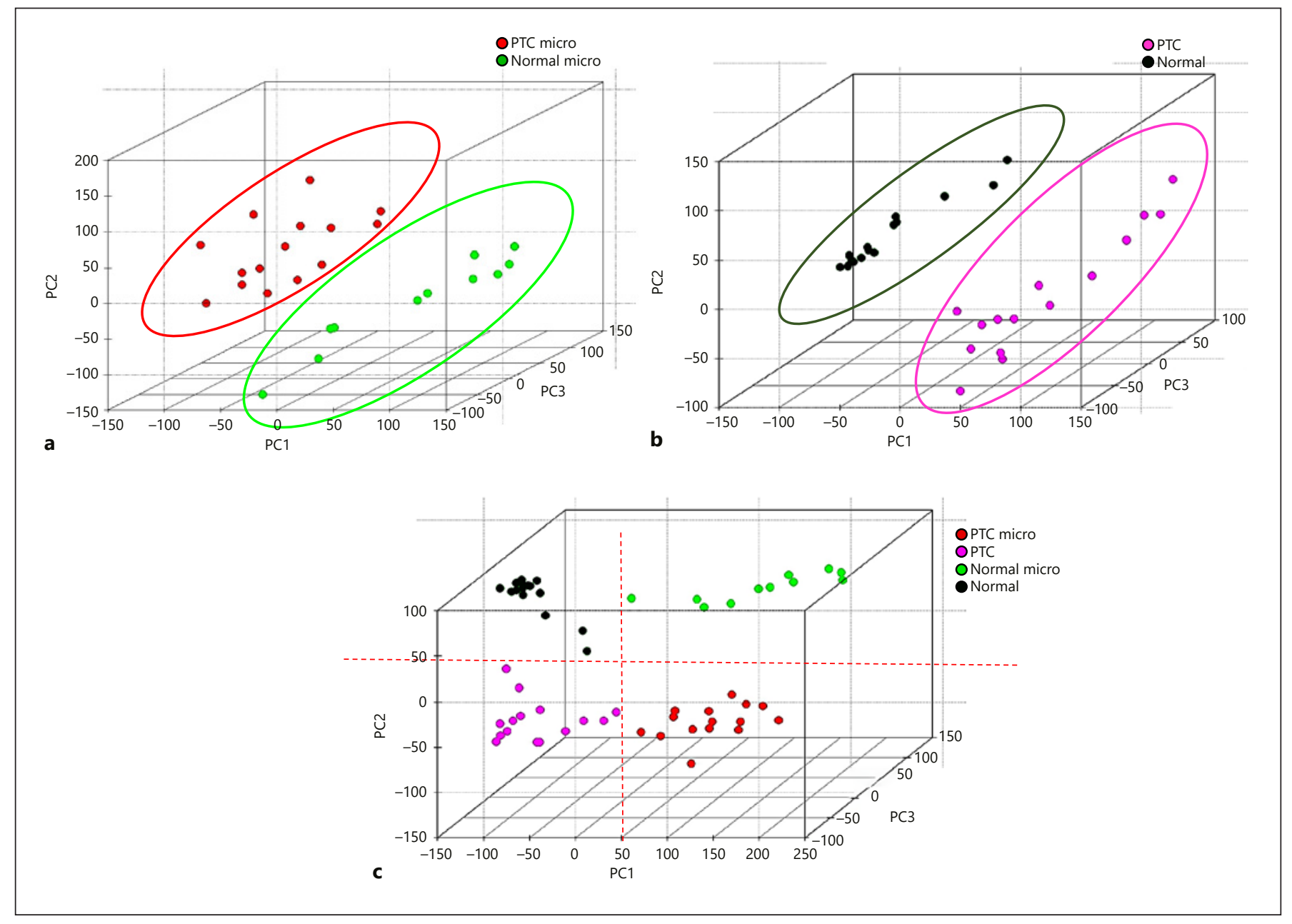

Fig. 1. Principal component analysis showed that the main variability in gene expression profile was related to sample used (microdissected cells vs. whole slide) (a, b) and to sample origin (PTC vs. normal thyroid regardless of material used - microdissected cells or whole slide) (c). PC, principal component; PTC, papillary thyroid cancer.

metric test was used for association of Kyoto Encyclopedia of Genes and Genomes (KEGG) biological pathways.

$q R T-P C R$ Analysis. Normalized qRT-PCR data were assessed by the nonparametric Mann-Whitney $U$ test adjusted by Bonferroni correction.

\section{Results}

In the first step, in order to assess the main source of variation, principal component analysis was performed separately in two groups: in a set containing thyrocytes obtained by microdissection and in a set containing whole slides of PTC tumor and normal thyroid (Fig. 1a, b). In both groups the main source of variation was the difference in gene expression profile between PTC samples and normal thyroid samples. Principal component analysis carried out in a combined set containing both microdissected cells and whole slides also revealed the difference in the transcriptome between PTC and normal thyroid. However, the differences between microdissected cells and whole slides were also visible. These differences were shown in the first principal component (Fig. 1c).

In the second step, supervised analysis was carried out in the following comparisons to select: (1) transcripts differentiating PTC and normal thyroid in microdissected cells and whole tissue slides; (2) transcripts differentiating PTC and normal thyroid in microdissected cells and not changed in whole tissue slides; (3) transcripts differentiating PTC and normal thyroid in the whole slides and not changed in microdissected cells. 


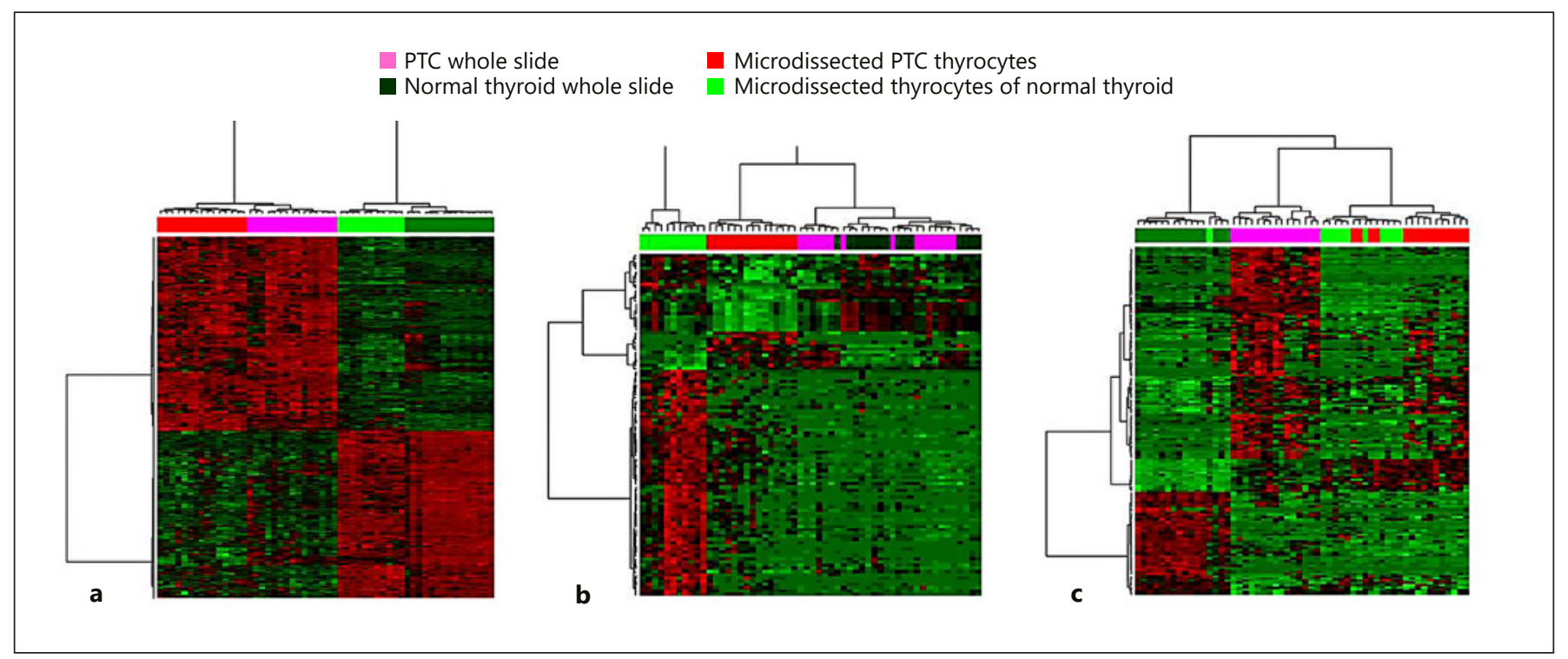

Fig. 2. Hierarchical clustering performed on the transcripts differentiating PTC and normal thyroid in microdissected cells and whole slides (a), in microdissected cells only (b), and in whole slides only (c). PTC, papillary thyroid cancer.

Table 1. Transcripts selected for qRT-PCR validation

\begin{tabular}{|c|c|c|}
\hline Transcript & $\begin{array}{l}\text { Microdissected } \\
\text { PTC cells }\end{array}$ & $\begin{array}{l}\text { Whole PTC } \\
\text { tumor slide }\end{array}$ \\
\hline \multicolumn{3}{|l|}{ Transcripts dysregulated in microdissected cells and whole slides } \\
\hline Trefoil factor 3 (TFF3) & downregulated & downregulated \\
\hline Fibronectin $1(F N 1)$ & upregulated & upregulated \\
\hline Metallophosphoesterase (MPPED2) & downregulated & downregulated \\
\hline Receptor MET & upregulated & upregulated \\
\hline Potassium inwardly-rectifying channel, subfamily J member (KCNJ2) & upregulated & upregulated \\
\hline Tumor-associated calcium signal transducer 2 (TACSTD2) & upregulated & upregulated \\
\hline UDP-galactose-4-epimerase (GALE) & upregulated & upregulated \\
\hline \multicolumn{3}{|l|}{ Transcripts dysregulated only in PTC microdissected cells } \\
\hline FBJ murine osteosarcoma viral oncogene homolog (FOS) (p55) & downregulated & ns \\
\hline Papillary thyroid carcinoma susceptibility candidate (PTCSC1) & downregulated & ns \\
\hline Connective tissue growth factor $(C T G F)$ & downregulated & ns \\
\hline Early growth response 1 (EGR1) & downregulated & ns \\
\hline
\end{tabular}

ns, not significant; PTC, papillary thyroid cancer; qRT-PCR, quantitative real-time polymerase chain reaction.

The first part of the supervised analysis identified 558 transcripts showing significant differences in expression between PTC and normal thyroid both in microdissected samples and whole slides (Fig. 2a; $p<0.001$, FDR $<2 \%$, and fold change $>4$ in both directions).

The goal of the second part of the supervised analysis was to select genes differentially expressed in the whole sections (nominal $p<0.01$, FDR $<2 \%$, and fold change $>4$ in both directions) and not altered in microdissected cells $(p>0.01)$. A total of 159 significantly deregulated transcripts were identified (Fig. 2b).

The third step of supervised analysis focused on the selection of transcripts with dysregulated expression only in microdissected cells (nominal $p<0.01, \mathrm{FDR}>2 \%$, and 


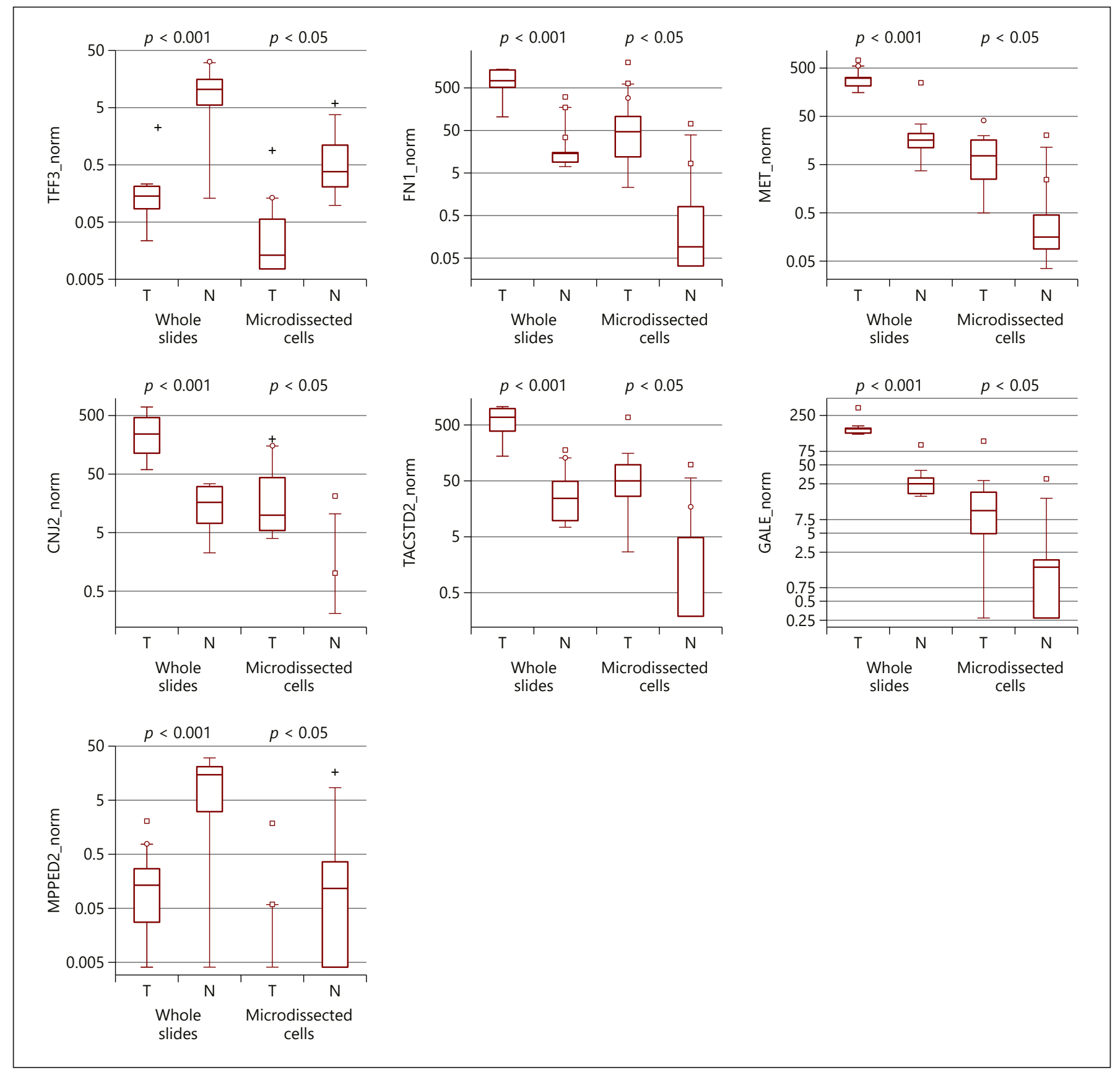

Fig. 3. Validation of seven genes differentially expressed between PTC and normal thyroid in both laser microdissected cells and whole slides in an independent set of samples. T, N, normal thyroid; PTC, papillary thyroid cancer; T, tumor.

fold change $>4$ in both directions) but not significant in whole tissue slides $(p>0.01)$. This analysis identified 106 differentially expressed transcripts (Fig. 2c).

Hierarchical clusterization of transcripts selected in a group combined of microdissected cells and whole tissue slides correctly separated PTC and normal thyroid sam- ples regardless of the sample analyzed. However, clusterization of differentiating transcripts obtained in the analyses of whole slides or microdissected cells alone separated samples in an incomplete manner (Fig. 2).

The last part of the study aimed to validate obtained microarray data. It was performed with the use of qRT- 

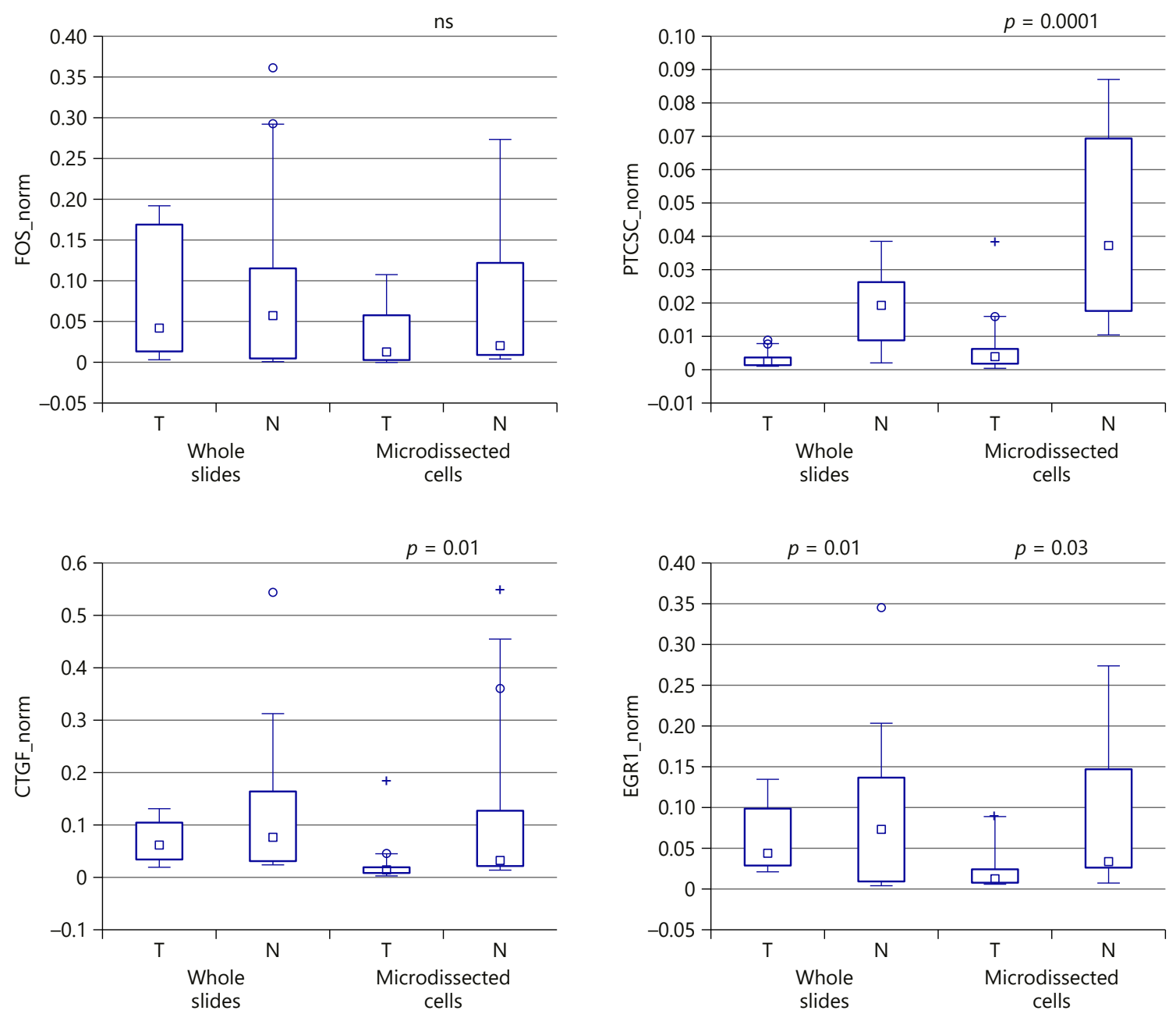

Fig. 4. Expression of four genes differentiating between PTC and normal thyroid only in microdissected cells, validated by qRT-PCR. N, normal thyroid; ns, not significant; PTC, papillary thyroid cancer; qRT-PCR, quantitative real-time polymerase chain reaction; $\mathrm{T}$, tumor.

PCR on an independent set of samples. Eleven transcripts were selected for qRT-PCR validation, among them seven transcripts differentially expressed between PTC and normal thyroid in both groups (microdissected cells and the whole slides) and four transcripts significantly differentiating PTC and normal thyroid only in microdissected cells (Table 1).

All seven selected genes demonstrating significant differences in expression between PTC and normal thyroid were confirmed by qRT-PCR (Fig. 3). Only one among four transcripts differentially expressed between PTC and normal thyroid in microdissected cells was not positively validated by qRT-PCR (FOS). Three other genes (PTCSC1, CTGF, EGFR1) were confirmed on an independent set of samples. In addition, the EGFR1 gene showed a significant difference in expression between PTC and normal thyroid in whole slides (Fig. 4).

All three transcript sets were also analyzed by the KEGG concerning biological pathways to determine possible implications of transcripts associated with microdissected cells and whole tissue slides. The pathways characteristic for each comparison are presented in Table 2. 
Table 2. KEGG biological pathways significant in PTC: microdissected cells and whole slides, only in microdissected cells, and only in whole slides

Transcripts differently expressed in
microdissected and whole slides
$p<0.001($ FDR $<2 \%)$, fold change $>4$ metabolism of xenobiotics by cytochrome P450

tyrosine metabolism

cell adhesion molecules

focal adhesion

ECM-receptor interaction

pathways in cancer

drug metabolism - cytochrome P450

axon guidance

colorectal cancer

small-cell lung cancer

complement and coagulation cascades

cytokine-cytokine receptor interaction

arrhythmogenic right ventricular cardiomyopathy

Transcripts differentially expressed in microdissected samples and not changed in whole slides

$p<0.001(\mathrm{FDR}<2 \%)$, fold change $>4$

vascular smooth muscle contraction

calcium signaling pathway

PPAR signaling pathway

GnRH signaling pathway

Transcripts differentially expressed in whole

slides and not changed in microdissected samples

$p<0.001(\mathrm{FDR}<2 \%)$, fold change $>4$

ECM, extracellular matrix; GnRH, gonadotropin-releasing hormone; KEGG, Kyoto Encyclopedia of Genes and Genomes; PPAR, peroxisome proliferator-activated receptor; PTC, papillary thyroid cancer.

\section{Discussion}

The evaluation of the transcriptome of selected transformed neoplastic thyrocytes and whole PTC slide compared to follicles of normal thyroid gland and macroscopically unchanged thyroid tissue demonstrated that the main source of variation was the difference between the whole section and the cell subjected to microdissection, while the differences in gene expression profile between neoplastic samples and normal thyroid remained significant. It means that the gene expression we observed during the analyses of the whole section was related to other cells than neoplastic ones. It has also been demonstrated that stromal cells interact with neoplastic cells. It is evidenced by the fact that genes selected in a set of microdissected cells and in a set of the whole slides allowed for a correct classification of PTC cells and normal thyroid cells, regardless of the analyzed material - microdissected cells or whole slide. Finally, eleven genes were selected to be verified in an independent set of samples; among them only four genes differentiated microdissected neoplastic cells and normal cells. Two genes, whose expression was significantly lower in PTC cells (PTCSC1 and CTGF), were confirmed in an independent validation set. Regarding two other genes, FOS (FBJ murine osteosarcoma viral oncogene homolog) was not confirmed by the validation, whereas for early growth response 1 (EGR1) a significant result was also obtained in the analysis of the whole slides.

The remaining genes (TFF3, FN1, MPPED2, MET, KCNJ2, TACSTD2, and GALE) showed varied expression in microdissected thyrocytes and in the whole tumor slides. It is worth noticing that these genes are frequently involved in classifiers characterizing PTC profile $[3,4,8]$.

The papillary thyroid carcinoma susceptibility candidate 1 gene (PTCSC1) is a noncoding RNA belonging to a group of long noncoding RNA, $>200$ nucleotides in length [23]. Recent studies demonstrated that long noncoding RNA could function both as oncogenes or suppressor genes [23].

The PTCSC1 gene is localized in a 3 intron of the Srclike adapter (SLA) gene (sense strand), which is in turn found in an intron of thyroglobulin gene (antisense strand) [24, 25]. In 2009, He et al. [24] proposed the PTCSC1 gene as a potential candidate gene related to a 
predisposition to PTC. This study also demonstrated lower expression of this gene in the majority of samples obtained from members of the family with a frequent occurrence of PTC. However, the authors did not find any mutation in a gene sequence which could be linked with predisposition to PTC or its lower expression in PTC.

The connective tissue growth factor gene (CTGF/ CCN2) belongs to the CNN gene family. It encodes a mitogen (mitosis-promoting factor) involved in cell division, differentiation, and cell adhesion [26]. The gene plays an essential role in the biology of connective tissue. It is expressed in most of the stromal cells (fibroblasts, endothelial cells, smooth muscle cells), stimulates the production of extracellular matrix (ECM) [27], and participates in wound healing. Its high expression is observed in stromal cells adjacent to different epithelial neoplasms, including breast or prostate cancer [26]. It is believed that the CTGF gene is a marker of wound healing and also a factor related to the progression of neoplastic disease. The study, carried out on a mouse prostate cancer xenograft model, showed that expression of the CTGF gene was related to the TME, that it played a crucial role in tumorreactive stroma, and that it was a stroma-related factor enhancing carcinogenesis [26].

The EGFR1 gene encodes a nuclear transcription regulatory protein. Trinh et al. [28] demonstrated that a decreased expression of this gene in tissues under hypoxic conditions promoted angiogenesis. EGFR1 may be regulated both by the MAPK/ERK pathway and hypoxia-inducible factor-1a (HIF-1), which activated EGFR1 promoter. The activation of MAPK pathway is characteristic for PTC [29], whereas the activation of the HIF-1 gene under hypoxic conditions is frequently noticed in solid tumors, among them thyroid cancer [30]. Angiogenesis is essential for tumor growth and progression, which is probably reflected by MAPK activation observed in PTC. Similarly, hypoxia leads to activation of proangiogenic factors, including the EGFR1 gene.

The remaining genes (TFF3, FN1, MPPED2, MET, TACSTD2, KCNJ2, and GALE), whose expression characterized the whole PTC slide and microdissected neoplastic transformed thyrocytes, reflect the reaction of the TME. The majority of them (MET, FN1, KCNJ2, TACSTD2, and GALE) showed significantly increased expression in normal thyroid tissue. The MET gene is a protooncogene encoding receptor tyrosine kinase which plays a role in signal transduction from the ECM to the cytoplasm [31]. Activation of the MET receptor is a consequence of a binding to hepatocyte growth factor (HGF) ligand. HGF ligand is a stromal-derived factor secreting by fibroblasts of the TME. Matsumoto et al. [31] demonstrated in 1989 that the TME was necessary to activate the MET receptor. The Japanese group also proved that fibroblasts were crucial for the invasion of oral squamous cell cancer cells and that HGF ligand was a factor responsible for this process $[31,32]$. Cell activation by stromal fibroblasts via stimulation of the MET receptor was also noticed in other malignancies, including breast, prostate, esophageal, and gallbladder carcinomas [33-36]. Moreover, the MET receptor showed a higher activity in hypoxic areas of the tumor. It could be related to a higher invasiveness of tumors showing a high level of hypoxia [37]. This dependence was also noticed in PTC. A high activity of MET protein was more pronounced at the tumor periphery, in regions of cell invasion promoted by HIF-1 [38].

Similar cancer-stromal interactions concern the expression of the fibronectin 1 gene (FN1). This gene encodes fibronectin, an ECM glycoprotein secreted by fibroblasts and present on the cell surface of fibroblasts or isolated from human plasma (fibronectin circulating in human plasma derives from hepatocytes). By binding to collagen, receptors on the cell surface (integrins), plasma fibrin, and glycosaminoglycans (heparin), FN1 is involved in cell adhesion and activates cell migration $[39,40]$. It could be expressed by epithelial cells [41]. High expression of the FN1 gene is frequently observed in PTC. Following the results of different studies concerning PTC transcriptome, the FN1 gene has been proposed as a molecular PTC marker in numerous papers $[3,42-45]$. However, attempts to use FN1 protein in immunostaining were not so successful. Expression of FN1 protein is observed in up to $40 \%$ of benign follicular lesions. Better results are obtained if the expression of several proteins is evaluated at the same time [46]. Tumor-stroma crosstalk, including FN1 protein expression, was also analyzed in melanoma and breast carcinoma $[47,48]$. These studies demonstrated an increased synthesis of FN1 protein and collagen and their release to the ECM by stromal cells, such as myofibroblasts [47, 48]. Moreover, it is believed that stromal fibroblasts, stimulated by the tumor cells, secrete different factors promoting tumor progression and growth [49]. The presence of FN1 protein in epithelial cells is also linked to the so-called epithelial-mesenchymal transition process, based on changes in biochemical and biological phenotype of epithelial cells, playing an essential role in physiology and pathology, including cancer progression. The transition of epithelial cells to mesenchymal ones leads to loss of cell-cell adhesion, cell polarity, and acquisition of migratory and invasive properties $[50,51]$. 
Another gene characterized by a significantly higher expression in the whole slide and selected PTC cells was tumor-associated calcium signal transducer 2 (TACSTD2). First data concerning its function pointed to its potential role as an oncogene [52]. Its expression was observed in different neoplasms, including PTC. However, according to recent data, the expression of the TACSTD2 gene was also noticed in normal, healthy cells [53], whereas in neoplastic cells, the TACSTD2 gene did not demonstrate oncogenic activity, but was related to the pro-cancer effect of the TME and epithelial-mesenchymal transition [54].

The potassium inwardly-rectifying channel, subfamily J member gene (KCNJ2) encodes Kir2.1 protein, a potassium channel that allows $\mathrm{K}^{+}$to move into the cell. In physiology, Kir2.1 protein is present in the majority of healthy cells, including neurons, muscle cells, and the immunological system. It is also expressed by neoplastic cells [55]. The UDP-galactose-4-epimerase gene (GALE) encodes UDP-galactose-4-epimerase that converts UDP-galactose to UDP-glucose. Its overexpression in PTC was confirmed by our previous study [43].

Significantly decreased expression of the trefoil factor 3 gene (TFF3) was also reported in other studies. Takano and Yamada [56], who analyzed the gene expression profile in thyroid tumors, proposed TFF3 as a marker of malignancy. Expression of this gene was observed in normal thyroid tissue, benign follicular neoplasms, and minimally invasive follicular carcinoma, whereas its decreased expression was characteristic for PTC. The authors tried to evaluate the expression of the protein encoded by TFF3 in immunostaining, but precise assessment of the intensity of such a reaction was not possible.

Decreased expression of the metallophosphoesterase domain containing 2 gene (MPPED2) in the whole PTC slide was demonstrated for the first time in our previous study [43]. MPPED2 was reported as a top gene which allowed for a proper classification of benign and malignant thyroid tumors in $96.7 \%$ of samples. This gene encodes a metallophosphoesterase and demonstrated high expression in the brain during mammal fetal development. New data regarding its potential role as a suppressor gene substantially involved in the neoplastic transformation of follicular thyroid cells have been published recently [57].

To date, only one study using whole-transcriptome profiling in PTC cells selected by laser microdissection has been reported [58]. However, one should stress that the comparison of gene expression between PTC cells and thyrocytes obtained from macroscopically unchanged thyroid tissue was not based on any statistical tests con-

Microinviroment in Papillary Thyroid Cancer sidering significance level or multiple-comparison correction. The authors selected up- or downregulated genes based on an expression ratio $>5.0$ or $<0.2$ in at least $50 \%$ of informative cases. There are no data regarding the number of analyzed microdissected cells. The FN1 gene was listed as the first among all genes commonly upregulated in thyroid cancer. The authors reported overexpression of $K C N J 2$, whereas the FOS gene was downregulated.

One should notice that most analyses carried out with the use of microdissected cells regarding the thyroid focused on the evaluation of single molecular markers [5963], whereas our approach allows for a more comprehensive look at the PTC transcriptome and surrounding microenvironment composed of different cells and ECM.

It is currently believed that cancer progression is promoted by the orchestrated interaction between malignant neoplastic cells and their environment. This interaction is, in some part, related to molecular alterations of mutated genes, which specifically drive the composition of the TME. It is widely accepted that dynamic crosstalk between the TME and tumor cells is involved in cancer progression, metastatic spread, recurrence, and response to drugs applied [64]. However, most studies which analyze the contribution of TME to cancer progression concern its single component and mainly focus on immune response $[65,66]$.

The crosstalk between the tumor and its microenvironment might also be visible in pathways activated by transcripts significantly differing in PTC microdissected cells and whole slides. The top pathway was "metabolism of xenobiotics by cytochrome P450," which mainly related to drug metabolism in cells affected by the TME, while the top KEGG pathway characterized for genes deregulated only in microdissected cells was "vascular muscle contraction." It refers to vascular smooth muscle cells that in pathological conditions may transfer to a proliferative state [67]. Regarding genes differentiating PTC and normal tissue observed only in whole tissue slide, the top KEGG pathway was "complement and coagulation cascades," crucial for the inflammatory network [68]. It could be related to immune response as an initial phase of a neoplastic transformation in the interaction between the tumor and its microenvironment.

For a better understanding of gene interactions between tumor cells and microenvironment, in the present study, we compared the gene expression profiles of PTC and normal thyroid in microdissected cells and whole tissue slides. To our best knowledge, this is the first study aimed at identifying and describing transcripts interacting between PTC and other cells composing the TME. We 
hope that our results on one hand reflect the complexity of tumor-microenvironment crosstalk and on the other hand contribute to a better understanding of PTC biology.

To conclude, most of the identified genes differentiating PTC and normal thyroid are related to the tumormicroenvironment interaction. One should answer the question of whether analysis of selected cancer cells is sufficient to know tumor biology or whether analysis of the whole tumor may better characterize tumor molecular profile.

\section{Statement of Ethics}

All subjects participating in this study gave their written informed consent and the study protocol was approved by the institute's committee on human research.

\section{Disclosure Statement}

The authors have no conflicts of interest to declare.

\section{Funding Sources}

The study was supported by the Polish National Center of Research and Development MILESTONE project - Molecular diagnostics and imaging in individualized therapy for breast, thyroid and prostate cancer, grant no. STRATEGMED 2/267398/4/ NCBR/2015.

\section{Author Contributions}

M. Oczko-Wojciechowska: study concept, study supervision, evaluation of gene expression profiles, data analysis, writing and correction of the manuscript. A. Pfeifer: statistical analyses. M. Jarzab: study concept, data analysis. M. Swierniak: statistical analyses. D. Rusinek: microdissection. T. Tyszkiewicz: qPCR validation. M. Kowalska: evaluation of gene expression profiles. E. Chmielik and E. Zembala-Nozynska: histopathological analysis. A. Czarniecka: surgical procedures, tumor sampling. B. Jarzab: study concept, study supervision, data analysis. J. Krajewska: study concept, study supervision, writing and correction of the manuscript. All authors discussed the results and contributed to the final version of the manuscript.

\section{References}

1 Haugen BR, Alexander EK, Bible KC, Doherty G, Mandel SJ, Nikiforov YE, et al. 2015 American Thyroid Association Management Guidelines for Adult Patients with Thyroid Nodules and Differentiated Thyroid Cancer: The American Thyroid Association Guidelines Task Force on Thyroid Nodules and Differentiated Thyroid Cancer. Thyroid. 2016; 26(1):1-133.

2 Rusinek D, Szpak-Ulczok S, Jarzab B. Gene expression profile of human thyroid cancer in relation to its mutational status. J Mol Endocrinol. 2011 Nov;47(3):R91-103.

3 Jarzab B, Wiench M, Fujarewicz K, Simek K, Jarzab M, Oczko-Wojciechowska M, et al. Gene expression profile of papillary thyroid cancer: sources of variability and diagnostic implications. Cancer Res. 2005 Feb;65(4): 1587-97.

4 Agrawal N, Akbani R, Aksoy BA, Ally A, Arachchi H, Asa SL, et al.; Cancer Genome Atlas Research Network. Integrated genomic characterization of papillary thyroid carcinoma. Cell. 2014 Oct;159(3):676-90.

5 Niemeier LA, Kuffner Akatsu H, Song C, Carty SE, Hodak SP, Yip L, et al. A combined molecular-pathologic score improves risk stratification of thyroid papillary microcarcinoma. Cancer. 2012 Apr;118(8):2069-77.

6 Han J, Chen M, Wang Y, Gong B, Zhuang T, Liang L, et al. Identification of Biomarkers Based on Differentially Expressed Genes in Papillary Thyroid Carcinoma. Sci Rep. 2018 Jul;8(1):9912.
7 Liu Y, Gao S, Jin Y, Yang Y, Tai J, Wang S, et al. Bioinformatics analysis to screen key genes in papillary thyroid carcinoma. Oncol Lett. 2020 Jan;19(1):195-204.

8 Huang Y, Prasad M, Lemon WJ, Hampel H, Wright FA, Kornacker K, et al. Gene expression in papillary thyroid carcinoma reveals highly consistent profiles. Proc Natl Acad Sci U S A. 2001 Dec;98(26):15044-9.

9 Salvatore G, Giannini R, Faviana P, Caleo A, Migliaccio I, Fagin JA, et al. Analysis of BRAF point mutation and RET/PTC rearrangement refines the fine-needle aspiration diagnosis of papillary thyroid carcinoma. J Clin Endocrinol Metab. 2004 Oct;89(10):5175-80.

10 Cheung CC, Carydis B, Ezzat S, Bedard YC, Asa SL. Analysis of ret/PTC gene rearrangements refines the fine needle aspiration diagnosis of thyroid cancer. J Clin Endocrinol Metab. 2001 May;86(5):2187-90.

11 Xing M, Tufano RP, Tufaro AP, Basaria S, Ewertz M, Rosenbaum E, et al. Detection of BRAF mutation on fine needle aspiration biopsy specimens: a new diagnostic tool for papillary thyroid cancer. J Clin Endocrinol Metab. 2004 Jun;89(6):2867-72.

12 Hayashida N, Namba H, Kumagai A, Hayashi $\mathrm{T}$, Ohtsuru A, Ito $\mathrm{M}$, et al. A rapid and simple detection method for the BRAF(T1796A) mutation in fine-needle aspirated thyroid carcinoma cells. Thyroid. 2004 Nov;14(11):9105 .
13 Domingues R, Mendonça E, Sobrinho L, Bugalho MJ. Searching for RET/PTC rearrangements and BRAF V599E mutation in thyroid aspirates might contribute to establish a preoperative diagnosis of papillary thyroid carcinoma. Cytopathology. 2005 Feb;16(1):27-31.

14 Cohen Y, Rosenbaum E, Clark DP, Zeiger MA, Umbricht CB, Tufano RP, et al. Mutational analysis of BRAF in fine needle aspiration biopsies of the thyroid: a potential application for the preoperative assessment of thyroid nodules. Clin Cancer Res. 2004 Apr; 10(8):2761-5.

15 Xing M, Alzahrani AS, Carson KA, Shong YK, Kim TY, Viola D, et al. Association between BRAF V600E mutation and recurrence of papillary thyroid cancer. J Clin Oncol. 2015 Jan;33(1):42-50.

16 Xing M, Alzahrani AS, Carson KA, Viola D, Elisei R, Bendlova B, et al. Association between BRAF V600E mutation and mortality in patients with papillary thyroid cancer. JAMA. 2013 Apr;309(14):1493-501.

17 Melo M, Gaspar da Rocha A, Batista R, Vinagre J, Martins MJ, Costa G, et al. TERT, BRAF, and NRAS in Primary Thyroid Cancer and Metastatic Disease. J Clin Endocrinol Metab. 2017 Jun;102(6):1898-907.

18 Melo M, da Rocha AG, Vinagre J, Batista R, Peixoto J, Tavares C, et al. TERT promoter mutations are a major indicator of poor outcome in differentiated thyroid carcinomas. J Clin Endocrinol Metab. 2014 May;99(5): E754-65. 
19 Privat-Maldonado A, Bengtson C, Razzokov J, Smits E, Bogaerts A. Modifying the Tumour Microenvironment: Challenges and Future Perspectives for Anticancer Plasma Treatments. Cancers (Basel). 2019 Dec;11(12):1920.

20 Roma-Rodrigues C, Mendes R, Baptista PV, Fernandes AR. Targeting Tumor Microenvironment for Cancer Therapy. Int J Mol Sci. 2019 Feb;20(4):840.

21 De Palma M, Biziato D, Petrova TV. Microenvironmental regulation of tumour angiogenesis. Nat Rev Cancer. 2017 Aug;17(8): $457-74$.

22 Lim B, Woodward WA, Wang X, Reuben JM, Ueno NT. Inflammatory breast cancer biology: the tumour microenvironment is key. Nat Rev Cancer. 2018 Aug;18(8):485-99.

23 Koirala P, Huang J, Ho TT, Wu F, Ding X, Mo YY. LncRNA AK023948 is a positive regulator of AKT. Nat Commun. 2017 Feb;8(1): 14422.

24 He H, Nagy R, Liyanarachchi S, Jiao H, Li W, Suster S, et al. A susceptibility locus for papillary thyroid carcinoma on chromosome 8q24. Cancer Res. 2009 Jan;69(2):625-31.

25 Mansha M, Carlet M, Ploner C, Gruber G, Wasim M, Wiegers GJ, et al. Functional analyses of Src-like adaptor (SLA), a glucocorticoid-regulated gene in acute lymphoblastic leukemia. Leuk Res. 2010 Apr;34(4):529-34.

26 Yang F, Tuxhorn JA, Ressler SJ, McAlhany SJ, Dang TD, Rowley DR. Stromal expression of connective tissue growth factor promotes angiogenesis and prostate cancer tumorigenesis. Cancer Res. 2005 Oct;65(19):8887-95.

27 Frazier K, Williams S, Kothapalli D, Klapper H, Grotendorst GR. Stimulation of fibroblast cell growth, matrix production, and granulation tissue formation by connective tissue growth factor. J Invest Dermatol. 1996 Sep; 107(3):404-11.

28 Trinh NT, Yamashita T, Ohneda K, Kimura K, Salazar GT, Sato F, et al. Increased Expression of EGR-1 in Diabetic Human Adipose Tissue-Derived Mesenchymal Stem Cells Reduces Their Wound Healing Capacity. Stem Cells Dev. 2016 May;25(10):760-73.

29 Jin S, Borkhuu O, Bao W, Yang YT. Signaling Pathways in Thyroid Cancer and Their Therapeutic Implications. J Clin Med Res. 2016 Apr;8(4):284-96.

30 Burrows N, Resch J, Cowen RL, von Wasielewski R, Hoang-Vu C, West CM, et al. Expression of hypoxia-inducible factor 1 alpha in thyroid carcinomas. Endocr Relat Cancer. 2010 Jan; 17(1):61-72.

31 Matsumoto K, Horikoshi M, Rikimaru K, Enomoto S. A study of an in vitro model for invasion of oral squamous cell carcinoma. J Oral Pathol Med. 1989 Oct;18(9):498-501.

32 Matsumoto K, Matsumoto K, Nakamura T, Kramer RH. Hepatocyte growth factor/scatter factor induces tyrosine phosphorylation of focal adhesion kinase (p125FAK) and promotes migration and invasion by oral squamous cell carcinoma cells. J Biol Chem. 1994 Dec;269(50):31807-13.
33 Matsumoto K, Date K, Shimura H, Nakamura T. Acquisition of invasive phenotype in gallbladder cancer cells via mutual interaction of stromal fibroblasts and cancer cells as mediated by hepatocyte growth factor. Jpn J Cancer Res. 1996 Jul;87(7):702-10.

34 Nakamura T, Matsumoto K, Kiritoshi A, Tano Y, Nakamura T. Induction of hepatocyte growth factor in fibroblasts by tumor-derived factors affects invasive growth of tumor cells: in vitro analysis of tumor-stromal interactions. Cancer Res. 1997 Aug;57(15):330513.

35 Date K, Matsumoto K, Kuba K, Shimura H, Tanaka M, Nakamura T. Inhibition of tumor growth and invasion by a four-kringle antagonist (HGF/NK4) for hepatocyte growth factor. Oncogene. 1998 Dec;17(23):3045-54.

36 Matsumoto-Taniura N, Matsumoto K, Nakamura T. Prostaglandin production in mouse mammary tumour cells confers invasive growth potential by inducing hepatocyte growth factor in stromal fibroblasts. Br J Cancer. 1999 Sep;81(2):194-202.

37 Pennacchietti S, Michieli P, Galluzzo M, Mazzone M, Giordano S, Comoglio PM. Hypoxia promotes invasive growth by transcriptional activation of the met protooncogene. Cancer Cell. 2003 Apr;3(4):347-61.

38 Scarpino S, Cancellario d'Alena F, Di Napoli A, Pasquini A, Marzullo A, Ruco LP. Increased expression of Met protein is associated with up-regulation of hypoxia inducible factor-1 (HIF-1) in tumour cells in papillary carcinoma of the thyroid. J Pathol. 2004 Mar; 202(3):352-8.

39 Jones TR, Ruoslahti E, Schold SC, Bigner DD. Fibronectin and glial fibrillary acidic protein expression in normal human brain and anaplastic human gliomas. Cancer Res. 1982 Jan; 42(1):168-77.

40 Zhang B, Shen S, Liao Z, Shi W, Wang Y, Zhao $\mathrm{J}$, et al. Targeting fibronectins of glioma extracellular matrix by CLT1 peptide-conjugated nanoparticles. Biomaterials. 2014 Apr;35(13): 4088-98.

41 Xiao J, Yang W, Xu B, Zhu H, Zou J, Su C, et al. Expression of fibronectin in esophageal squamous cell carcinoma and its role in $\mathrm{mi}$ gration. BMC Cancer. 2018 Oct;18(1):976.

42 Griffith OL, Melck A, Jones SJ, Wiseman SM. Meta-analysis and meta-review of thyroid cancer gene expression profiling studies identifies important diagnostic biomarkers. J Clin Oncol. 2006 Nov;24(31):5043-51.

43 Fujarewicz K, Jarzab M, Eszlinger M, Krohn K, Paschke R, Oczko-Wojciechowska M, et al. A multi-gene approach to differentiate papillary thyroid carcinoma from benign lesions: gene selection using support vector machines with bootstrapping. Endocr Relat Cancer. 2007 Sep;14(3):809-26.

44 Finley DJ, Arora N, Zhu B, Gallagher L, Fahey TJ 3rd. Molecular profiling distinguishes papillary carcinoma from benign thyroid nodules. J Clin Endocrinol Metab. 2004 Jul;89(7): 3214-23.
45 Hawthorn L, Stein L, Varma R, Wiseman S, Loree T, Tan D. TIMP1 and SERPIN-A overexpression and TFF3 and CRABP1 underexpression as biomarkers for papillary thyroid carcinoma. Head Neck. 2004 Dec;26(12): 1069-83.

46 Liu YY, Morreau H, Kievit J, Romijn JA, Carrasco N, Smit JW. Combined immunostaining with galectin-3, fibronectin-1, CITED-1, Hector Battifora mesothelial-1, cytokeratin-19, peroxisome proliferator-activated receptor-\{gamma\}, and sodium/iodide symporter antibodies for the differential diagnosis of non-medullary thyroid carcinoma. Eur J Endocrinol. 2008 Mar;158(3):375-84.

47 Li G, Satyamoorthy K, Meier F, Berking C, Bogenrieder T, Herlyn M. Function and regulation of melanoma-stromal fibroblast interactions: when seeds meet soil. Oncogene. 2003 May;22(20):3162-71.

48 Noel A, Kebers F, Maquoi E, Foidart JM. Cellcell and cell-matrix interactions during breast cancer progression. Curr Top Pathol. 1999; 93:183-93.

49 Mueller MM, Fusenig NE. Friends or foes bipolar effects of the tumour stroma in cancer. Nat Rev Cancer. 2004 Nov;4(11):839-49.

50 Thiery JP, Acloque H, Huang RY, Nieto MA. Epithelial-mesenchymal transitions in development and disease. Cell. 2009 Nov; 139(5): $871-90$

51 Kalluri R, Weinberg RA. The basics of epithelial-mesenchymal transition. J Clin Invest. 2009 Jun;119(6): 1420-8

52 Wang J, Day R, Dong Y, Weintraub SJ, Michel L. Identification of Trop-2 as an oncogene and an attractive therapeutic target in colon cancers. Mol Cancer Ther. 2008 Feb;7(2): 280-5.

53 McDougall AR, Tolcos M, Hooper SB, Cole TJ, Wallace MJ. Trop2: from development to disease. Dev Dyn. 2015 Feb;244(2):99-109.

54 Wang J, Zhang K, Grabowska D, Li A, Dong Y, Day R, et al. Loss of Trop2 promotes carcinogenesis and features of epithelial to mesenchymal transition in squamous cell carcinoma. Mol Cancer Res. 2011 Dec;9(12):168695.

55 Hibino H, Inanobe A, Furutani K, Murakami S, Findlay I, Kurachi Y. Inwardly rectifying potassium channels: their structure, function, and physiological roles. Physiol Rev. 2010 Jan; 90(1):291-366

56 Takano T, Yamada H. Trefoil factor 3 (TFF3) a promising indicator for diagnosing thyroid follicular carcinoma. Endocr J. 2009;56(1): 9-16.

57 Sepe R, Pellecchia S, Serra P, D’Angelo D, Federico A, Raia M, et al. The Long Non-Coding RNA RP5-1024C24.1 and Its AssociatedGene MPPED2 Are Down-Regulated in $\mathrm{Hu}-$ man Thyroid Neoplasias and Act as Tumour Suppressors. Cancers (Basel). 2018 May; 10(5):146. 
58 Nikolova DN, Zembutsu H, Sechanov T, Vidinov K, Kee LS, Ivanova R, et al. Genomewide gene expression profiles of thyroid carcinoma: identification of molecular targets for treatment of thyroid carcinoma. Oncol Rep. 2008 Jul;20(1):105-21.

59 McCarthy RP, Wang M, Jones TD, Strate RW, Cheng L. Molecular evidence for the same clonal origin of multifocal papillary thyroid carcinomas. Clin Cancer Res. 2006 Apr;12(8): 2414-8.

60 Gasbarri A, Sciacchitano S, Marasco A, Papotti M, Di Napoli A, Marzullo A, et al. Detection and molecular characterisation of thyroid cancer precursor lesions in a specific subset of Hashimoto's thyroiditis. Br J Cancer. 2004 Sep;91(6):1096-104.

61 Finn S, Smyth P, O’Regan E, Cahill S, Toner $\mathrm{M}$, Timon C, et al. Low-level genomic insta- bility is a feature of papillary thyroid carcinoma: an array comparative genomic hybridization study of laser capture microdissected papillary thyroid carcinoma tumors and clonal cell lines. Arch Pathol Lab Med. 2007 Jan; 131(1):65-73.

62 Bongiovanni $\mathrm{M}$, Molinari F, Eszlinger $\mathrm{M}$, Paschke R, Barizzi J, Merlo E, et al. Laser capture microdissection is a valuable tool in the preoperative molecular screening of follicular lesions of the thyroid: an institutional experience. Cytopathology. 2015 Oct;26(5):288-96.

63 Tallini G, Brandao G. Assessment of RET/ PTC oncogene activation in thyroid nodules utilizing laser microdissection followed by nested RT-PCR. Methods Mol Biol. 2005;293: 103-11.

64 Guo S, Deng CX. Effect of Stromal Cells in Tumor Microenvironment on Metastasis Ini- tiation. Int J Biol Sci. 2018 Nov;14(14):208393.

65 Cunha LL, Marcello MA, Ward LS. The role of the inflammatory microenvironment in thyroid carcinogenesis. Endocr Relat Cancer. 2014 Apr;21(3):R85-103.

66 Ferrari SM, Fallahi P, Galdiero MR, Ruffilli I, Elia G, Ragusa F, et al. Immune and Inflammatory Cells in Thyroid Cancer Microenvironment. Int J Mol Sci. 2019 Sep;20(18): 4413.

67 Touyz RM, Alves-Lopes R, Rios FJ, Camargo LL, Anagnostopoulou A, Arner A, et al. Vascular smooth muscle contraction in hypertension. Cardiovasc Res. 2018 Mar;114(4):52939.

68 Rittirsch D, Flierl MA, Ward PA. Harmful molecular mechanisms in sepsis. Nat Rev Immunol. 2008 Oct;8(10):776-87. 